\title{
Repeated Sirolimus-Eluting Stent Implantation to Treat Sirolimus-Eluting Stent and Bare-Metal Stent Restenosis
}

\author{
Kensaku Nishihira, MD; Yoshisato Shibata, MD; Tetsunori Ishikawa, MD; \\ Katsumasa Nomura, MD; Tatsuya Nakama, MD; Daigo Mine, MD; Yohei Inoue, MD; \\ Keiichi Ashikaga, MD; Nehiro Kuriyama, MD; Akihiko Matsuyama, MD; \\ Takuroh Imamura, MD; Yujiro Asada, MD; Kazuo Kitamura, MD
}

\begin{abstract}
Background: In-stent restenosis (ISR) remains a persistent, unresolved issue even in the era of percutaneous coronary intervention (PCl) using drug-eluting stents. The present study compares the clinical and angiographic outcomes of using sirolimus-eluting stents (SES) for re-intervention against ISR that was originally treated with sirolimus-eluting or bare-metal (BMS) stents.
\end{abstract}

Methods and Results: This prospective single-center registry investigated 179 ISR lesions in 158 consecutive patients (53 lesions in 49, and 126 in 109 patients originally treated with SES and BMS, respectively), who had undergone re-intervention with SES. The patients were clinically and angiographically followed up at 8 months after re-PCl. The incidence of re-restenosis (29 vs $12 \%, \mathrm{P}<0.01$ ), ischemia-driven target lesion revascularization (TLR; 21 vs $8 \%, \mathrm{P}<0.05$ ) and major adverse cardiac events (MACE; 21 vs $9 \%, \mathrm{P}<0.05$ ) were significantly greater in ISR lesions originally treated with SES than in those originally treated with BMS at 8 months after re-PCI. Moreover, late luminal loss was significantly greater in the group with post-SES restenosis $(P<0.05)$. Even after adjustment, post-SES restenosis was the only independent predictor of re-restenosis and $M A C E(P<0.05$, each).

Conclusions: Although the re-restenosis rate is acceptable, the incidence rates of late restenosis, ischemiadriven TLR and MACE are higher after repeated SES implantation to treat SES, than BMS restenosis. These results might affect the mid-term clinical outcomes of re-intervention with SES. (Circ J 2010; 74: 2329-2333)

Key Words: Cardiovascular diseases; Coronary re-intervention; Ischemia; Revascularization

A lthough drug-eluting stents (DES) have significantly decreased the incidence of in-stent restenosis (ISR) and the need for repeated revascularization compared with bare-metal stents (BMS), ${ }^{1-5}$ DES restenosis still develops and ISR remains an important clinical issue especially for patients with highly complex lesions. ${ }^{2-7}$ The increasing use of DES in complex settings coupled with the worldwide implantation of $>10$ million DES $^{6}$ implies that DES restenosis will become a significant global problem. However, an optimal treatment for DES restenosis remains unknown, and some experts propose repeated DES implantation. In contrast, several studies have shown the effectiveness of DES in patients with BMS ISR.4,5 A recent study suggested different pathological features between intra-DES and intra-BMS restenotic tissue. ${ }^{8}$ These 2 types of ISR lesion might have different biological responses and clinical outcomes after DES implantation. We therefore compared the clinical and angiographic outcomes of re-intervention with sirolimus-eluting stents (SES; Cypher, Cordis/Johnson \& Johnson, Warren, NJ, USA) for ISR lesions that were originally treated with SES or BMS.

\section{Methods}

\section{Study Patients}

The study population of this prospective single-center registry comprised 158 consecutive patients (179 lesions) who underwent re-intervention with SES for ISR between August 2004 and June 2007. Among them, restenosis developed in 49 patients with SES (53 lesions) and in 109 with BMS (126

Received March 8, 2010; revised manuscript received June 28, 2010; accepted July 7, 2010; released online September 28, 2010 Time for primary review: 43 days

Department of Cardiology, Miyazaki Medical Association Hospital, Miyazaki (K. Nishihira, Y.S., K. Nomura, T.N., D.M., Y.I., K.A., N.K., A.M.); Division of Circulatory and Body Fluid Regulation, Department of Internal Medicine (K. Nishihira, T. Ishikawa, A.M., T. Imamura, K.K.), Department of Pathology, Faculty of Medicine (Y.A.), University of Miyazaki, Miyazaki, Japan

Mailing address: Kensaku Nishihira, MD, PhD, Department of Cardiology, Miyazaki Medical Association Hospital, 738-1 Funado, Shinbeppu-cho, Miyazaki 880-0834, Japan. E-mail: nishihira@fc.miyazaki-u.ac.jp

ISSN-1346-9843 doi:10.1253/circj.CJ-10-0210

All rights are reserved to the Japanese Circulation Society. For permissions, please e-mail: cj@j-circ.or.jp 


\begin{tabular}{|c|c|c|c|}
\hline Patients (n) & SES $(n=49)$ & BMS $(n=109)$ & $P$ value \\
\hline Age (years) & $67 \pm 8.3$ & $66.6 \pm 10.8$ & 0.88 \\
\hline Male gender, n (\%) & $41(84)$ & $92(84)$ & $>0.99$ \\
\hline Previous MI, n (\%) & $20(41)$ & $65(60)$ & 0.04 \\
\hline Previous CABG, n (\%) & $1(2)$ & $5(5)$ & 0.75 \\
\hline \multicolumn{4}{|l|}{ Risk factor, n (\%) } \\
\hline Hypertension & $36(73)$ & $80(73)$ & $>0.99$ \\
\hline Hyperlipidemia & $28(57)$ & $42(41)$ & 0.09 \\
\hline Current smoking & $6(12)$ & $19(17)$ & 0.55 \\
\hline Diabetes mellitus & $28(57)$ & $36(33)$ & $<0.01$ \\
\hline
\end{tabular}

Data are expressed as mean \pm SD when appropriate.

SES, sirolimus-eluting stent; BMS, bare-metal stent; MI, myocardial infarction; CABG, coronary artery bypass graft.

\begin{tabular}{|c|c|c|c|}
\hline Target lesion (n) & SES $(n=53)$ & BMS $(n=126)$ & $P$ value \\
\hline Target vessel, $\mathbf{n}(\%)$ & & & 0.32 \\
\hline Left anterior descending & $19(36)$ & $57(45)$ & \\
\hline Left circumflex & $13(24)$ & $20(16)$ & \\
\hline Right coronary artery & $21(40)$ & $49(39)$ & \\
\hline In-stent restenosis type, $\mathbf{n}(\%)^{\star}$ & & & $<0.01$ \\
\hline \multicolumn{4}{|l|}{ Focal } \\
\hline 1 & $36(68)$ & $25(20)$ & \\
\hline \multicolumn{4}{|l|}{ Non-focal } \\
\hline ॥ & $11(21)$ & $65(52)$ & \\
\hline III & $5(9)$ & $29(23)$ & \\
\hline IV & $1(2)$ & $7(5)$ & \\
\hline \multicolumn{4}{|l|}{ Pre-procedure } \\
\hline Reference diameter (mm) & $2.66 \pm 0.54$ & $2.66 \pm 0.61$ & 0.97 \\
\hline MLD (mm) & $0.58 \pm 0.37$ & $0.74 \pm 0.41$ & 0.02 \\
\hline Diameter stenosis $(\%)$ & $77.9 \pm 13.9$ & $71 \pm 17.6$ & 0.01 \\
\hline Lesion length $(\mathrm{mm})$ & $17 \pm 6.2$ & $21 \pm 8.1$ & $<0.01$ \\
\hline Post-procedure MLD (mm) & $2.68 \pm 0.48$ & $2.79 \pm 0.51$ & 0.18 \\
\hline
\end{tabular}

Data are expressed as mean \pm SD when appropriate.

MLD, minimal lumen diameter. Other abbreviations see in Table 1.

${ }^{*}$ According to the classification by Mehran et al. ${ }^{9}$

lesions). Patients were eligible for the study if they had initial ISR in a native coronary artery with objective evidence of ischemia and without clinical contraindication against prolonged double antiplatelet therapy. All patients provided written, informed consent to participate in the study, and our institutional ethics committee approved the study protocol. We defined ISR using quantitative coronary angiography (QCA) as luminal stenosis of $>50 \%$ within the stent or within $5 \mathrm{~mm}$ of the stent edges. The type of restenosis was categorized as focal (length $<10 \mathrm{~mm}$ ) and non-focal (diffuse, proliferative and occlusive) according to the classification of Mehran et al. ${ }^{9}$

Procedural anticoagulation therapy included heparin targeted to an activated clotting time of 200 to 300 s. All patients underwent repeated percutaneous coronary intervention (rePCI) with SES according to current guidelines, and the choice of the implanted PCI devices was left to the operators' discretion. Then, if possible, intravascular ultrasound (IVUS)-guided re-PCI was performed to rule out a possible mechanism of stent failure such as underexpansion and to confirm neointimal growth in ISR lesions. All patients received aspirin ( 100 or $200 \mathrm{mg} /$ day) before, and indefinitely after the pro- cedure. Patients were also concomitantly treated with ticlopidine $(200 \mathrm{mg} /$ day $)$ or clopidogrel $(50 \mathrm{mg} / \text { day or } 75 \mathrm{mg} / \text { day })^{10}$ for at least 8 months. The patients were followed up angiographically at 8 months post re-PCI or earlier if non-invasive evaluation or clinical presentation suggested ischemia.

\section{Quantitative Coronary Angiography}

Coronary angiograms were analyzed using a validated edge detection system (CMS, MEDIS, Leiden, The Netherlands) by 2 experienced cardiologists (K.N. and T.N.) who were blinded to the clinical classification of the patients. Minimal lumen diameter (MLD), reference vessel diameter, and $\%$ diameter stenosis at baseline, post procedure and at follow up were measured. Angiographic re-restenosis was defined by QCA as stenosis of $>50 \%$ diameter within a previously stented segment (within the stent or $5 \mathrm{~mm}$ of the stent edges) on 8-month follow-up angiograms. Late luminal loss was defined as the difference between MLD at the time of the post stenting procedure and that at follow up.

\section{Clinical Follow up}

Patients were followed up to assess the incidence of major 


\begin{tabular}{|c|c|c|c|}
\hline Target lesion (n) & $\begin{array}{c}\text { SES group } \\
(n=53)\end{array}$ & $\begin{array}{l}\text { BMS group } \\
(n=125)\end{array}$ & $P$ value \\
\hline \multicolumn{4}{|l|}{ Follow up } \\
\hline Reference diameter $(\mathrm{mm})$ & $2.89 \pm 0.45$ & $2.93 \pm 0.54$ & 0.66 \\
\hline $\operatorname{MLD}(\mathrm{mm})$ & $2.01 \pm 1.08$ & $2.41 \pm 0.91$ & 0.01 \\
\hline Diameter stenosis $(\%)$ & $30.3 \pm 33.6$ & $17.9 \pm 26.6$ & 0.02 \\
\hline Angiographic re-restenosis, $n(\%)$ & $16(29)$ & $15(12)$ & $<0.01$ \\
\hline TLR, n (\%) & $11(21)$ & $10(8)$ & 0.02 \\
\hline TVR, n (\%) & $11(21)$ & $11(9)$ & 0.04 \\
\hline MACE, n (\%) & $11(21)$ & $11(9)$ & 0.04 \\
\hline Stent thrombosis, $\mathrm{n}(\%)$ & $2(4)$ & $1(1)$ & 0.16 \\
\hline
\end{tabular}

Data are expressed as mean \pm SD when appropriate.

TLR, target lesion revascularization; TVR, target vessel revascularization; MACE, major adverse cardiac event. Other abbreviations see in Tables 1 and 2.

adverse cardiac events (MACE) including all-cause death, myocardial infarction (MI), target lesion revascularization (TLR) and target vessel revascularization (TVR). We defined $\mathrm{MI}$ as the presence of a new $\mathrm{Q}$ wave in $\geq 2$ contiguous ECG leads and/or a creatine kinase-MB concentration elevated to $>3$-fold the upper normal limit. We defined TLR as repeated re-intervention to treat an ISR accompanied by symptoms or objective signs of ischemia (ischemia-driven TLR). We then defined TVR as any repeated revascularization procedure involving a target vessel with an ischemic sign (ischemic-driven TVR). Stent thrombosis was defined according to the designation established by the Academic Research Consortium. ${ }^{11}$

\section{Statistical Analysis}

Data are expressed as mean \pm SD. The differences between the 2 groups were analyzed using an unpaired Student's t-test or the Mann-Whitney U test when the variance was skewed. Categorical variables were compared by Fisher's exact probability test. We also assessed independent predictors of angiographic re-restenosis and MACE using multivariate logistic regression analysis with independent variables that were considered significant according to univariate analyses. All tests were 2 -sided and a $\mathrm{P}$ value of $<0.05$ was considered statistically significant.

\section{Results}

We studied a total of 179 ISR lesions in 158 consecutive patients (53 SES restenotic lesions in 49 patients, and $126 \mathrm{BMS}$ restenotic lesions in 109 patients) between August 2004 and June 2007. All patents were successfully implanted with SES and 178 patients $(99 \%)$ were followed up by angiography, for which 1 patient refused to undergo.

Tables 1 and 2 show baseline demographic, clinical, and angiographic characteristics and procedural variables. Patients with post-SES restenosis had a higher prevalence of diabetes mellitus and a lower prevalence of MI history than those with post-BMS restenosis (Table 1). Patients with SES restenosis also had more frequent focal restenosis, lesions with a significantly smaller MLD, a higher rate of \%diameter stenosis and shorter lesions than those with BMS restenosis (Table 2). No periprocedural MI, TLR or stent thrombosis or in-hospital deaths occurred.

Table 3 and Figure show the QCA data and clinical outcomes at 8 months of follow up. The MLD was significantly smaller and the rate of \%diameter stenosis was more increased in ISR lesions originally treated with SES than with

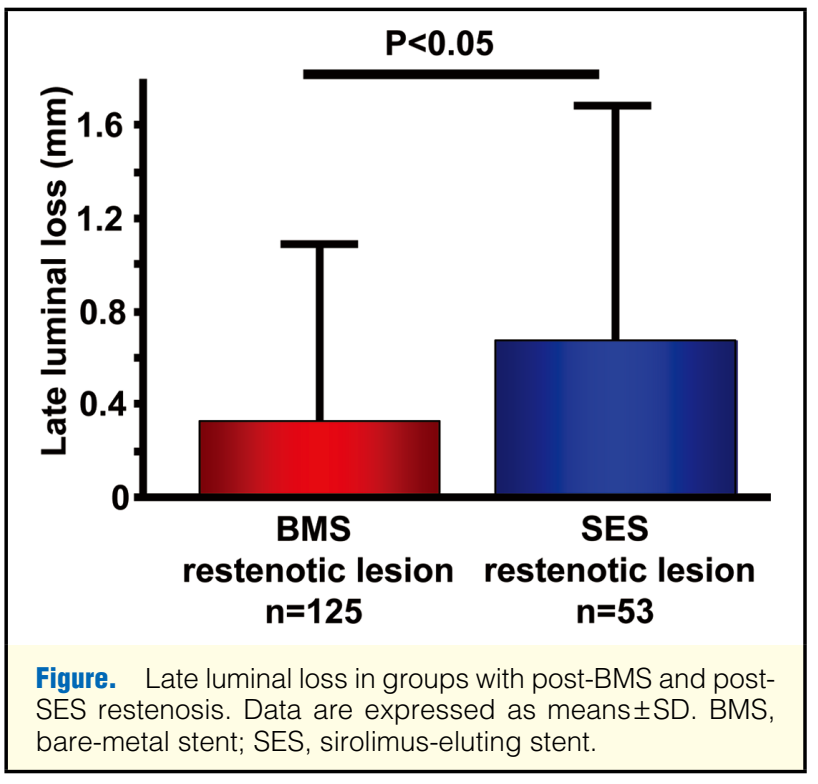

BMS. Interestingly, the rates of angiographic re-restenosis ( $29 \%$ vs $12 \%, \mathrm{P}<0.01)$, TLR ( $21 \%$ vs $8 \%, \mathrm{P}=0.02)$, TVR ( $21 \%$ vs $9 \%, \mathrm{P}=0.04)$ and $\mathrm{MACE}(21 \%$ vs $9 \%, \mathrm{P}=0.04)$ were significantly higher in the group with post-SES than postBMS restenosis (Table 3). Moreover, late luminal loss was significantly greater in the SES than in the BMS group $(0.68 \pm$ 1.01 vs $0.36 \pm 0.77 \mathrm{~mm}, \mathrm{P}=0.04$; Figure). The type of re-restenosis was generally focal in both groups (data not shown). No cardiac death and Q-wave MI occurred during follow up. Stent thrombosis resulting in unstable or stable angina pectoris developed in $1(1 \%)$ and $2(4 \%)$ patients in the BMS and SES groups, respectively, but the difference was not statistically significant.

Multivariate logistic regression analysis also showed that repeated SES implantation to treat restenosis of SES is an independent predictor of angiographic re-restenosis and MACE (odds ratio (OR) 3.4, 95\% confidence interval (CI) [1.28-9.02], $\mathrm{P}=0.01$ and $\mathrm{OR} 3.37$, 95\%CI [1.08-10.49], $\mathrm{P}=0.04$, respectively) compared with BMS, after including all variables that were significant in the univariate analyses listed in Tables 1 and 2 (Tables 4,5).

Also, the incidence of re-restenosis and MACE did not differ between patients with and without diabetes mellitus (19\% vs $16 \%, \mathrm{P}=0.7$ and $14 \%$ vs $11 \%, \mathrm{P}=0.78$, respectively). 


\begin{tabular}{|c|c|c|c|c|c|c|}
\hline & \multicolumn{3}{|c|}{ Univariate } & \multicolumn{3}{|c|}{ Multivariate } \\
\hline & OR & $95 \% \mathrm{Cl}$ & $P$ value & OR & $95 \% \mathrm{Cl}$ & $P$ value \\
\hline SES group & 3.2 & $1.44-7.1$ & $<0.01$ & 3.4 & $1.28-9.02$ & 0.01 \\
\hline Diabetes mellitus & 1.24 & $0.57-2.71$ & 0.59 & 0.92 & $0.4-2.12$ & 0.85 \\
\hline Previous MI & 0.68 & $0.31-1.47$ & 0.68 & 0.81 & $0.36-1.85$ & 0.62 \\
\hline \multicolumn{7}{|l|}{ Pre-procedure } \\
\hline MLD & 0.52 & $0.19-1.45$ & 0.21 & 0.48 & $0.08-2.91$ & 0.42 \\
\hline Lesion length & 0.96 & $0.91-1.02$ & 0.18 & 0.97 & $0.92-1.03$ & 0.36 \\
\hline$\%$ Diameter stenosis & 1.01 & $0.99-1.04$ & 0.34 & 0.99 & $0.95-1.04$ & 0.72 \\
\hline Lesion type: Focal & 1.28 & $0.57-2.84$ & 0.55 & 0.59 & $0.22-1.56$ & 0.29 \\
\hline
\end{tabular}

Variables were $\mathrm{P}<0.05$ in univariate analyses listed in Tables 1 and 2.

$\mathrm{OR}$, odds ratio; $\mathrm{Cl}$, confidence interval. Other abbreviations see in Tables 1 and 2.

\begin{tabular}{|c|c|c|c|c|c|c|}
\hline & \multicolumn{3}{|c|}{ Univariate } & \multicolumn{3}{|c|}{ Multivariate } \\
\hline & OR & $95 \% \mathrm{Cl}$ & $P$ value & OR & $95 \% \mathrm{Cl}$ & $P$ value \\
\hline SES group & 3.04 & $1.2-7.67$ & 0.02 & 3.37 & $1.08-10.49$ & 0.04 \\
\hline Diabetes mellitus & 1.37 & $0.55-3.42$ & 0.5 & 1.05 & $0.4-2.74$ & 0.92 \\
\hline Previous MI & 0.54 & $0.21-1.35$ & 0.19 & 0.61 & $0.23-1.61$ & 0.32 \\
\hline \multicolumn{7}{|l|}{ Pre-procedure } \\
\hline MLD & 0.83 & $0.26-2.59$ & 0.74 & 0.82 & $0.1-6.79$ & 0.86 \\
\hline Lesion length & 0.97 & $0.91-1.04$ & 0.43 & 0.99 & $0.92-1.05$ & 0.65 \\
\hline \% Diameter stenosis & 1 & $0.98-1.03$ & 0.8 & 0.99 & $0.94-1.04$ & 0.75 \\
\hline Lesion type: Focal & 1.22 & $0.48-3.12$ & 0.68 & 0.61 & $0.2-1.88$ & 0.39 \\
\hline
\end{tabular}

Variables were $\mathrm{P}<0.05$ in univariate analyses listed in Tables 1 and 2.

Abbreviations see in Tables 1-4.

\section{Discussion}

The main finding of the present study was that repeated SES implantation for post-SES restenosis although safe, was associated with relatively higher rates of recurrent restenosis and MACE than that for post-BMS restenosis at 8 months of follow up. We also discovered significantly greater late luminal loss in the SES group.

The implantation of SES has significantly decreased the incidence of restenosis and the need for TLR..$^{1-3}$ In the clinical environment, SES are increasingly being implanted into patients with various higher risk indications such as ISR, small vessels, chronic total occlusions coronary artery calcification, and diabetes. ${ }^{3-7,12,13}$ These applications have led to an observed rate of DES restenosis of $>5 \% .{ }^{3,6,7,12,13}$ More uses of SES in complex settings will probably increase the incidence of restenosis. Thus, SES restenosis will become an important issue even in the DES era. However, because of the relatively low incidence of SES restenosis, few studies have evaluated the effectiveness of repeated SES implantation for SES restenosis, and the clinical and angiographic outcomes of re-intervention with SES to treat restenosis of SES and BMS have not been determined. The present study addressed these issues. Several retrospective studies, ${ }^{6,10,14-17}$ have suggested higher restenosis and TLR rates after treating restenosis of various DES, including SES, than after treating de novo lesions. Furthermore, current therapies for DES ISR or DES thrombosis are associated with a high long-term rate of MACE (42.9\%). ${ }^{18}$ The present study prospectively discovered that repeated SES implantation to treat SES restenosis is associated with a higher incidence of late restenosis, ischemiadriven TLR and MACE than SES implantation to treat BMS restenosis. The rates of re-restenosis, TLR or MACE in the present study were relatively similar to the findings of several retrospective studies. ${ }^{6,10,14-17}$ Our results support previous findings and indicate that although SES is an excellent approach to treating patients with BMS restenosis, the same cannot be said for treating those with SES restenosis.

Local features, angiographic findings of restenosis type and diabetes mellitus might play important roles in post-SES restenosis. ${ }^{5-7,12}$ Lemos et al ${ }^{16}$ reported that recurrent SES restenosis is particularly high among patients with hypercholesterolemia, previous angioplasty, failed brachytherapy and post-SES restenosis treated with balloon dilation. Several investigators then also suggested that repeated SES implantation to treat non-focal SES restenosis and smaller-diameter vessels is an independent predictor of re-restenosis. ${ }^{10,17} \mathrm{How}-$ ever, multivariate logistic regression analysis showed that diabetes mellitus, type of restenosis on angiograms, lesion length and procedural MLD did not significantly influence the outcomes after repeated intervention in our series.

The mechanisms of the high rates of recurrent restenosis after repeated SES implantation for SES restenosis are poorly understood. Possible explanations include enhanced allergic or inflammatory responses to the polymer or the drug as well as a late catch-up phenomenon, excessive intimal hyperplastic responses that are inadequately blocked by the drug or the amount of drug delivered, stent fracture and stent underexpansion. ${ }^{6} 19-22$ In the present study, IVUS-guided PCI was performed to exclude underexpansion at the second intervention. In contrast, although the drug-resistance (sirolimusresistance) hypothesis seems attractive, no clinical studies have yet demonstrated a clear clinical benefit of implanting a DES that is different from the original., ${ }^{6,15}$ The small 
sample size of these retrospective non-randomized studies might have limited the statistical power to demonstrate differences or detect potential adverse effects. Further study is required to evaluate this issue.

The limitations of this study are as follows. First, although this is a prospective study with a high rate of angiographic follow up, the small sample size might not have been sufficient to reach a definitive conclusion. However, to recruit a large number of patients is complicated by low SES restenosis rates. Further investigations are required to confirm our observations. Second, this is a non-randomized analysis at a single center. Third, in this study, we did not compare the effect of repeated SES implantation with that of other possible therapies, including balloon angioplasty or implantation with a different DES. Recently, some investigators have reported the efficacy of repeated DES implantation for DES restenosis compared with conventional balloon angioplasty. ${ }^{10,23}$ In the preliminary study, we also assessed the rate of re-restenosis of balloon angioplasty for SES restenosis. The re-restenosis rate of balloon angioplasty for SES restenosis tended to be higher than in that of repeated SES implantation, but the difference did not reach significance (data not shown).

In conclusion, the incidence of late restenosis and MACE is higher after repeated SES implantation to treat restenosis of SES, than of BMS. These results might affect the midterm clinical outcomes of re-intervention with SES.

\section{Disclosure}

Conflict of interest: None declared.

\section{References}

1. Morice MC, Serruys PW, Sousa JE, Fajadet J, Ban Hayashi E, Perin M, et al. A randomized comparison of a sirolimus-eluting stent with a standard stent for coronary revascularization. $N$ Engl $J$ Med 2002; 346: $1773-1780$.

2. Moses JW, Leon MB, Popma JJ, Fitzgerald PJ, Holmes DR, O'Shaughnessy C, et al. Sirolimus-eluting stents versus standard stents in patients with stenosis in a native coronary artery. New Engl J Med 2003; 349: 1315-1323.

3. Ardissino D, Cavallini C, Bramucci E, Indolfi C, Marzocchi A, Manari A, et al. Sirolimus-eluting vs uncoated stents for prevention of restenosis in small coronary arteries: A randomized trial. JAMA 2004; 292: 2727-2734.

4. Dibra A, Kastrati A, Alfonso F, Seyfarth M, Perez-Vizcayno MJ, Mehilli J, et al. Effectiveness of drug-eluting stents in patients with bare-metal in-stent restenosis: Meta-analysis of randomized trials. J Am Coll Cardiol 2007; 49: 616-623.

5. Liistro F, Fineschi M, Angioli P, Sinicropi G, Falsini G, Gori T, et al. Effectiveness and safety of sirolimus stent implantation for coronary in-stent restenosis: The TRUE (Tuscany registry of sirolimus for unselected in-stent restenosis) registry. J Am Coll Cardiol 2006; 48: 270-275.

6. Aminian A, Kabir T, Eeckhout E. Treatment of drug-eluting stent restenosis: An emerging challenge. Catheter Cardiovasc Interv 2009; 74: $108-116$.

7. Cosgrave J, Melzi G, Biondi-Zoccai GGL, Airoldi F, Chieffo A, Sangiorgi GM, et al. Drug-eluting stent restenosis: The pattern pre- dicts the outcome. J Am Coll Cardiol 2006; 47: 2399-2404.

8. Chieffo A, Foglieni C, Nodari RL, Briguori G, Sangiorgi G, Latib A, et al. Histopathology of clinical coronary stenosis in drug-eluting versus bare metal stents. Am J Cardiol 2009; 104: 1660-1667.

9. Mehran R, Dangas G, Abizaid AS, Mintz GS, Lansky AJ, Satler LF, et al. Angiographic patterns of in-stent restenosis: Classification and implications for long-term outcome. Circulation 1999; 100: $1872-1878$

10. Kitahara H, Kobayashi Y, Takebayashi H, Fujimoto Y, Nakamura Y, Kuroda N, et al. Re-restenosis and target lesion revascularization after treatment of sirolimus-eluting stent restenosis: Retrospective analysis of 4 Japanese hospitals. Circ J 2009; 73: 867-871.

11. Mauri L, Hsieh WH, Massaro JM, Ho KK, D'Agostino R, Cutlip DE. Stent thrombosis in randomized clinical trials of drug-eluting stents. N Engl J Med 2007; 356: 1020 - 1029.

12. Lemos PA, Hoye A, Goedhart D, Arampatzis CA, Saia F, van der Giessen WJ, et al. Clinical, angiographic, and procedural predictors of angiographic restenosis after sirolimus-eluting stent implantation in complex patients: An evaluation from the rapamycin-eluting stent evaluated at Rotterdam Cardiology Hospital (RESEARCH) study. Circulation 2004; 109: 1366-1370.

13. Mori S, Yasuda S, Kataoka Y, Morii I, Kawamura A, Miyazaki S. Significant association of coronary artery calcification in stent delivery route with restenosis after sirolimus-eluting stent implantation. Circ J 2009; 73: 1856-1863.

14. Steinberg DH, Gaglia MA Jr, Pinto Slottow TL, Roy P, Bonello L, De Labriolle A, et al. Outcome differences with the use of drugeluting stents for the treatment of in-stent restenosis of bare-metal stents versus drug-eluting stents. Am J Cardiol 2009; 103: $491-$ 495.

15. Cosgrave J, Melzi G, Corbett S, Biondi-Zoccai GG, Babic R, Airoldi F, et al. Repeated drug-eluting stent implantation for drugeluting stent restenosis: The same or a different stent. Am Heart $J$ 2007; 153: 354-359.

16. Lemos PA, van Mieghem CA, Arampatzis CA, Hoye A, Ong AT, McFadden E, et al. Post-sirolimus-eluting stent restenosis treated with repeat percutaneous intervention: Late angiographic and clinical outcomes. Circulation 2004; 109: 2500-2502.

17. Chitani K, Muramatsu T, Tsukahara R, Ito Y, Ishimori H, Hirano $\mathrm{K}$, et al. Predictive factors of re-restenosis after repeated sirolimuseluting stent implantation for SES restenosis and clinical outcomes after percutaneous coronary intervention for SES restenosis. J Interven Cardiol 2009; 22: 354-361.

18. Mishkel GJ, Moore AL, Markwell S, Shelton MC, Shelton ME. Long-term outcomes after management of restenosis or thrombosis of drug-eluting stents. J Am Coll Cardiol 2007; 49: 181-184.

19. Fujii K, Mintz GS, Kobayashi Y, Carlier SG, Takebayashi H, Yasuda T, et al. Contribution of stent underexpansion to recurrence after sirolimus-eluting stent implantation for in-stent restenosis. Circulation 2004; 109: 1085-1088.

20. Finn AV, Kolodgie FD, Harnek J, Guerrero LJ, Acampado E, Tefera $\mathrm{K}$, et al. Differential response of delayed healing and persistent inflammation at sites of overlapping sirolimus- or paclitaxel-eluting stents. Circulation 2005; 112: 270-278.

21. Lim SY, Jeong MH, Hong SJ, Lim DS, Moon JY, Hong YJ, et al. Inflammation and delayed endothelization with overlapping drugeluting stents in a porcine model of in-stent restenosis. Circ J 2008; 72: $463-468$.

22. Ino Y, Toyoda Y, Tanaka A, Ishii S, Kusuyama Y, Kubo T, et al. Predictors and prognosis of stent fracture after sirolimus-eluting stent implantation. Circ J 2009; 73: 2036-2941.

23. Kim YH, Lee BK, Park DW, Park KH, Choi BR, Lee CW, et al. Comparison with conventional therapies of repeated sirolimus-eluting stent implantation for the treatment of drug-eluting coronary stent restenosis. Am J Cardiol 2006; 98: 1451 - 1454. 